\title{
Frontier between separability and quantum entanglement in a many spin system
}

\author{
Francisco C. Alcaraz ${ }^{(a)}$ and Constantino Tsallis ${ }^{(b, c)}$ \\ (a) Departamento de Física, Universidade Federal de São Carlos, Via Washington Luiz, São Carlos-SP, Brazil \\ (b) Centro Brasileiro de Pesquisas Físicas, Xavier Sigaud 150,22290-180, Rio de Janeiro-RJ, Brazil (tsallis@cbpf.br) \\ (c) Erwin Schroedinger International Institute for Mathematical Physics, Boltzmanngasse 9, A-1090 Wien, Austria
}

(November 20, 2018)

\begin{abstract}
We discuss the critical point $x_{c}$ separating the quantum entangled and separable states in two series of $N$ spins $S$ in the simple mixed state characterized by the matrix operator $\rho=x|\tilde{\phi}><\tilde{\phi}|+$ $\frac{1-x}{D^{N}} I_{D^{N}}$ where $x \in[0,1], D=2 S+1, \mathbf{I}_{D^{N}}$ is the $D^{N} \times D^{N}$ unity matrix and $\mid \tilde{\phi}>$ is a special entangled state. The cases $x=0$ and $x=1$ correspond respectively to fully random spins and to a fully entangled state. In the first of these series we consider special states $|\tilde{\phi}\rangle$ invariant under charge conjugation, that generalizes the $N=2 \operatorname{spin} S=\frac{1}{2}$ Einstein-Podolsky-Rosen state, and in the second one we consider generalizations of the Weber density matrices. The evaluation of the critical point $x_{c}$ was done through bounds coming from the partial transposition method of Peres and the conditional nonextensive entropy criterion. Our results suggest the conjecture that whenever the bounds coming from both methods coincide the result of $x_{c}$ is the exact one. The results we present are relevant for the discussion of quantum computing, teleportation and cryptography.
\end{abstract}

Quantum entanglement is a quite amazing physical phenomenon. It was first discussed in depth in 1935 by Einstein, Podolsky and Rosen (EPR) [1] and by Schroedinger [2], and since then by many others [3 20]. This phenomenon has attracted intensive interest in recent years due to its applications in quantum computation, teleportation and cryptography, as well as to its connections to quantum chaos. Special importance is attributed to the frontier which, in mixed states, separates the quantum entangled from the so called separable states. Separability, precisely defined in what follows, is essentially a classical concept, and it is the one on which the possibility of a description in terms of local realism with hidden variables is currently built on. The state of a system composed of subsystems $A$ and $B$ is said separable (or unentangled) if and only if its corresponding density matrix $\rho_{A+B}$ can be written as

$$
\rho_{A+B}=\sum_{i=1}^{W} p_{i} \rho_{A}^{(i)} \otimes \rho_{B}^{(i)} \quad\left(p_{i} \geq 0 \forall i ; \sum_{i=1}^{W} p_{i}=1\right)
$$

The frontier between quantum entangled and separable states for a variety of mixed states is currently discussed in the literature. Several necessary conditions for separability are already available; however, no general sufficient conditions are yet known. Among the existing necessary conditions two criteria have shown some preference in the literature due to their simplicity and the fact that in some cases these conditions coincide with the exact ones. Those are the Peres criterium [13] based on the positivity of the partially transposed density matrix, and the generalization of standard entropic methods, given by the conditional nonextensive entropy criterium, recently advanced by Abe and Rajagopal [21]. In the present paper we are going to apply and compare both methods by ap- plying them to several distinct density matrices coming from some many body systems. Both criteria recover exact separable-entangled frontiers in some simple mixed states of a two spin $1 / 2$ system 21,22, but only provide a bound in more complex cases. Although no rigorous proof is available, as we shall see, the analysis of some particular cases suggests that generically the partial transpose method overestimates the separable region either equally or less than the conditional entropy method. Moreover, our results indicate that whenever both methods yield the same result, this result is the exact one. It is clear that the discussion of quantum entanglement in many body systems is crucial both conceptually (e.g., for understanding the interface between the classical and quantum descriptions of the real world) and practically (e.g., for efficiently implementing the input and output of a device such as a quantum computer). Some specific $N$-body systems have already been discussed in the literature (see 23,24 for some classes of systems with arbitrary $N$, and [25] for the Greenberger-Horne-Zeilinger state of $N=3$ spins $1 / 2$; see 26] for a review).

Let us consider a $N$-body system composed by spin- $S$ operators acting on a Hilbert space $H=H_{1} \otimes \ldots \otimes H_{n}$ of dimension $D^{N}=(2 S+1)^{N}$. An arbitrary density matrix acting on this space should have all its eigenvalues non negative. Consider an arbitrary orthogonal basis $\mid s_{1}, \ldots, s_{N}>\left(s_{i}=-S, \ldots, S\right)$ spanning the Hilbert space. The Peres criterion appropriate to this $N$-body systems asserts that from a given density matrix $\rho$, with elements $<s_{1}, \ldots, s_{N}|\rho| s_{1}^{\prime}, \ldots, s_{N}^{\prime}>$, it is possible to define other density matrices $\sigma^{i_{1}, \ldots, i_{n}}$, where the spin coordinates $i_{1}, \ldots, i_{n}\left(i_{1}, \ldots, i_{n}=1, \ldots, N\right)$, are transposed ("in $\leftrightarrow$ out"), i. e., $<s_{1} \cdots s_{N}\left|\sigma^{i_{1}, \ldots, i_{n}}\right| s_{1}^{\prime} \cdots s_{N}^{\prime}>=$

$$
<s_{1} \cdots s_{i_{1}-1} s_{i_{1}}^{\prime} s_{i_{1}+1} \cdots s_{i_{2}}^{\prime} \cdots s_{i_{n}}^{\prime} s_{i_{n}+1} \cdots s_{N} \mid
$$




$$
\rho \mid s_{1}^{\prime} \cdots s_{i_{1}-1}^{\prime} s_{i_{1}} s_{i_{1}+1}^{\prime} \cdots s_{i_{2}} \cdots s_{i_{n}} s_{i_{n}+1}^{\prime} \cdots s_{N}^{\prime}>.
$$

The non-negativity of these new density matrices give us the Peres criterion, namely all eigenvalues of $\sigma^{i_{1}, \ldots, i_{n}}$ should be non-negative.

In order to probe and compare the above mentioned methods we will consider several sets of density matrices of general type

$$
\rho(x)=(1-x) \rho(0)+x \tilde{\rho}
$$

where $\rho(0)=\rho_{1} \otimes \ldots \otimes \rho_{N}=\mathbf{I}_{D^{N}} / D^{N}, \mathbf{I}_{D^{N}}$ being the $D^{N} \times D^{N}$ unity matrix, is a fully separable state and $\tilde{\rho}=|\tilde{\phi}><\tilde{\phi}|$ is a density matrix corresponding to a known entangled state $|\tilde{\phi}\rangle$. Then as we increase the value of $x$, there exists a critical value $x=x_{c}$ where the quantum entanglement takes place, and we want to obtain bounds for $x_{c}$.

The matrices $\sigma^{i_{1}, \ldots, i_{n}}$ obtained by the partial transposition at indexes $\left(i_{1}, \ldots, i_{n}\right)$, are given by

$$
\sigma^{i_{1}, \cdots, i_{n}}(x)=\frac{1-x}{D^{N}} \mathbf{I}_{D^{N}}+x \tilde{\sigma}^{i_{1}, \ldots, i_{n}}
$$

where $\quad<\quad s_{1}, \ldots, s_{N}\left|\tilde{\sigma}^{i_{1}, \ldots, i_{n}}\right| s_{1}^{\prime}, \ldots, s_{N}^{\prime} \quad>=<$ $s_{1}, \ldots, s_{i_{1}}^{\prime} \cdots s_{i_{2}}^{\prime} \cdots, s_{i_{n}}^{\prime}, \ldots, s_{N}|\tilde{\rho}| s_{1}^{\prime} \cdots s_{i_{1}} \cdots s_{i_{2}} \cdots s_{i_{n}}$ $\cdots s_{N}^{\prime}>$. The $D^{N}$ eigenvalues of $\sigma^{i_{1}, \ldots, i_{n}}(x)$ are given by $E_{i}=(1-x) / D^{N}+x \tilde{E}_{i}$, where $\tilde{E}_{i}\left(i=1, \ldots, D^{L}\right)$ are the eigenvalues of $\tilde{\sigma}^{i_{1}, \ldots, i_{n}}$. The condition for non-negative eigenvalues imply that quantum entanglement is present at least for $x \geq x_{c}^{P}$ ( $P$ stands for Peres), where

$$
x_{c}^{P}=\frac{1}{1-D^{N} \tilde{\tilde{E}}},
$$

and $\tilde{\tilde{E}}$ is the lowest eigenvalue of $\tilde{\sigma}^{i_{1}, \ldots i_{n}}$. In principle we should consider all the possible transpositions $i_{1}, \ldots, i_{n}$ but, in all the applications we considered, the transposition of a single spin, i. e., $\sigma^{i_{1}}$, gave us the best (i.e., the lowest) bound for $x_{c}$.

The second criterion we analyze was introduced recently by Abe and Rajagopal [21]. Exploring the classical composition of probabilities arising from the nonextensive entropic form on which nonextensive statistical mechanics [27] is based, they provide a condition for quantum unentanglement. Within this entropic approach the condition for quantum unentanglement is that the conditional probability for any subsystem forming the whole system should be non-negative. In this entropic approach the entropy of a $N$-spin $S$ system $A_{1}+\cdots A_{N}$, formed by subsystems $A_{i}(i=1, \ldots, N)$, with density $\rho_{A_{1}+\cdots A_{N}}$ is given by

$$
S_{q}\left(A_{1}+\cdots A_{N}\right)=\frac{1-\operatorname{Tr} \rho_{A_{1}+\cdots+A_{N}}^{q}}{q-1}
$$

where $q$ is an arbitrary real number. The particular case $q \rightarrow 1$ recovers the standard von Neumann entropy. The conditional entropies, taking into account a partial information of the system, are given by

$$
\begin{aligned}
& S_{q}\left(A_{1}+\cdots+A_{i_{1}-1}+A_{i_{1}+1}+\cdots+A_{i_{n}-1}+A_{i_{n}+1}+\right. \\
& \left.\cdots+A_{N} \mid A_{i_{1}}+A_{i_{2}}+\cdots+A_{i_{n}}\right)= \\
& \frac{S_{q}\left(A_{1}+\cdots+A_{N}\right)-S_{q}\left(A_{i_{1}}+A_{i_{2}}+\cdots+A_{i_{n}}\right)}{1+(1-q) S_{q}\left(A_{i_{1}}+A_{i_{2}}+\cdots+A_{i_{n}}\right)}
\end{aligned}
$$

where $S_{q}\left(A_{1}+\cdots+A_{n}\right)$ is given by (5) with

$$
\begin{aligned}
& \rho_{A_{i_{1}}+\cdots+A_{i_{n}}}= \\
& \operatorname{Tr}_{A_{1}, \ldots, A_{i_{1}-1}, A_{i_{1}+1}, \ldots, A_{i_{n-1}}, A_{i_{n+1}}, \ldots, A_{N}}\left[\rho_{A_{1}+\cdots+A_{N}}\right] .
\end{aligned}
$$

The condition for non-negative conditional probability imply that, for arbitrary combinations $\left\{i_{1}, \ldots, i_{n}\right\}$, (6) should be non-negative, and consequently the bound for $x_{c}$ is evaluated by imposing

$$
\operatorname{Tr} \rho_{A_{1}+\cdots A_{N}}^{q}=\operatorname{Tr} \rho_{A_{i_{1}}+\cdots+A_{i_{n}}}^{q} .
$$

The left side of the above equation is simple to calculate for the special set of density matrices (2) by going to the basis where $\tilde{\rho}$ is diagonal, and we obtain

$$
\operatorname{Tr} \rho_{A_{1}+\cdots+A_{n}}^{q}=\left(D^{N}-1\right)\left(\frac{1-x}{D^{N}}\right)^{q}+\left(\frac{1+\left(D^{N}-1\right) x}{D^{N}}\right)^{q} .
$$

From (2) and (7) we obtain

$$
\rho_{A_{i_{1}}+\cdots+A_{i_{n}}}=\frac{1-x}{D^{N}} D^{N-n} \mathbf{I}_{D^{n}}+x \tilde{\tilde{\rho}}_{A_{i_{1}}+\cdots+A_{i_{n}}},
$$

where

$$
\begin{aligned}
\tilde{\tilde{\rho}}_{A_{i_{1}}+\cdots+A_{i_{n}}}= & \operatorname{Tr}_{A_{i_{1}}, \ldots, A_{i_{1}-1}, A_{i_{1}+1}, \ldots, A_{i_{n}-1}, A_{i_{n}+1} \ldots, A_{N}} \\
& {\left[\tilde{\rho}_{A_{1}+\cdots+A_{N}}\right], }
\end{aligned}
$$

is a $D^{n} \times D^{n}$ matrix calculated from (2). If we denote by $v_{i}\left(i=1, \ldots, D^{n}\right)$ the eigenvalues of $\tilde{\tilde{\rho}}$ the relations (8)-(10) give us

$$
\begin{aligned}
& \left(D^{N}-1\right)\left(\frac{1-x}{D^{N}}\right)^{q}+\left(\frac{1+\left(D^{N}-1\right) x}{D^{N}}\right)^{q}= \\
& \sum_{i=1}^{D^{n}}\left(\frac{1-x}{D^{N}} D^{N-n}+x v_{i}\right)^{q} .
\end{aligned}
$$

This equation will give us $x_{c}(q)$ for each value of $q$. Due to the monotonic properties of the entropy (5), the lowest bound for $x_{c}$ is obtained in the limiting case $q \rightarrow \infty$. In this limit we can neglect the first term in the left hand side of (12) and keep in the sum only the term corresponding to the largest eigenvalue $\bar{v}(N)$ of the matrix $\tilde{\tilde{\rho}}_{A_{i_{1}}+\cdots+A_{i_{n}}}$, which gives

$$
x_{c}^{S}=\frac{1}{1+\frac{D^{N}}{D^{N-n}-1}(1-\bar{v})},
$$


where the superscript refers to entropy. In principle we should consider the conditional probabilities of arbitrary subsystems. In our applications the largest eigenvalue, i.e., the one which produces the most restrictive bound, is obtained when we consider, in these conditional probabilities, the subsystem $A_{1}+\ldots+A_{N-1}$.

We are going to consider initially density matrices written in vector basis invariant under charge conjugation, or spin-reversal symmetry. In the $S^{z}$-basis the basis vectors are given by the non-null combinations $\mid \phi_{i}^{c}>=\frac{1}{\sqrt{2}}\left(\left|s_{1}, \ldots, s_{N}>+c\right|-s_{1}, \ldots,-s_{N}>\right),(i=$ $\left.1, \ldots, D^{N}, c= \pm 1\right)$. For $S=\frac{1}{2}$ and $N=2$ this basis recovers the standard Bell basis. We now consider the density matrix $\rho(x)$ given by (2) where $\tilde{\rho}=\left|\phi_{k}^{-}><\phi_{k}^{-}\right|$is formed by an arbitrary basis vector with charge conjugation eigenvalue $c=-1$, i. e., $\mid \phi_{k}^{-}>=(|1>-| 2>) / \sqrt{2}$, with $|1\rangle=\mid s_{1}, \ldots, s_{N}>$ and $|2\rangle=\left|-s_{1}, \ldots,-s_{N}\right\rangle$ $\left(\left\{s_{i}\right\}\right.$ arbitrary). It is simple to convince ourselves that in the standard basis the only non-zero elements of $\tilde{\rho}$ in (2) are given by $\langle 1|\tilde{\rho}| 1>=<2|\tilde{\rho}| 2\rangle=-<1|\tilde{\rho}| 2\rangle=$ $-<2|\tilde{\rho}| 1>=1 / 2$. In order to apply the Peres criterion we consider the density matrix $\tilde{\sigma}$ defined in (3) by the transposition of the last spin. The only non-zero terms of the related matrix $\tilde{\sigma}$ are given by $<1|\tilde{\sigma}| 1>=<$ $2|\tilde{\sigma}| 2>=1 / 2,<3|\tilde{\sigma}| 4>=<1|\tilde{\rho}| 2>=<3|\tilde{\sigma}| 4>=<$ $2|\tilde{\rho}| 1>=-1 / 2$, where $|3>=| s_{1}, \ldots, s_{N-1},-s_{N}>$ and $|4>=|-s_{1}, \ldots,-s_{N-1}, s_{N}>$. This last matrix has the lowest eigenvalue $-1 / 2$; consequently, from (何), we get the bound

$$
x_{c}=x_{c}^{P}=\frac{1}{1+D^{N} / 2},
$$

for all values of $D$ and $N$.

In the application of the entropic bound, the most restrictive condition happens when we consider the conditional probability of the subsystem composed by $(N-1)$ spins and it is not difficult to see that $\tilde{\tilde{\rho}}$ in (10) has only two nonzero elements $(=1 / 2)$ in the diagonal, that gives the eigenvalue $\bar{v}=1 / 2$ and from (13) the bound

$$
x_{c}^{S}=\frac{1}{1+\frac{D^{N}}{2} \frac{1}{D-1}} .
$$

Comparing (14) and (15) we see that for $S=1 / 2$ both criteria give us the same bound $x_{c}^{P}=x_{c}^{S}=\frac{1}{1+2^{N-1}}$, but for general values of the spin $S \neq 1 / 2$ the bound coming from Peres criterion is more restrictive than that of the entropic criterion since $x_{c}^{P}<x_{c}^{S}$. It is interesting to remark that in the case $S=1 / 2$, where the bounds coincide the value is known to be the exact value for $x_{c}$, for $N=2$ [12] or $N>2$ [28]. The bounds (14) give us an interesting result. Suppose we need, as would occur in a quantum computer, to couple the special entangled state $\mid \phi_{k}>$ with a white spectrum environment. The mixed system will be described by the density matrix given by (2), where the parameter $x$ controls the coupling with the environment. The bound (14) tell us that we keep the whole system entangled as long as the ratio between the component of our special vector $\mid \phi_{k}>$ and any other vector in the environment, forming the density matrix, is larger than $\left(1+\left(D^{N}-1\right) x_{c}^{P}\right) /\left(1-x_{c}^{P}\right)$ that as $N \rightarrow \infty$ tend towards 3 , independently of the value of $\mathrm{S}$.

A second class of $N$ spins $\mathrm{S}$ density matrices we consider is a generalization of the Werner density matrices [29], given by (2) with

$$
\left|\tilde{\phi}>=\frac{1}{\mathcal{N}} \sum_{k=-S}^{S} a_{k}\right| k, k, \ldots, k>, \quad \mathcal{N}=\sum_{k=-S}^{S}\left|a_{k}\right|^{2} .
$$

The standard spin- $S$ Werner density matrix, corresponds to $a_{-S}=\cdots=a_{S}$ and it was shown [28] that for this case the exact value where quantum entanglement takes place is $x_{c}=1 /\left(1+D^{N-1}\right)$. For this particular case it was also shown recently [24] that the entropic bound (13) also coincides with the above exact value $x_{c}^{S}=x_{c}$. The application of the Peres criterion by transposing the last spin give us the eigenvalues $\pm\left|a_{l}^{*} a_{k}\right| / \mathcal{N}(l \neq k)$ or $\left|a_{k}\right|^{2} / \mathcal{N}$ for the matrix $\tilde{\sigma}$ in (3) and consequently the bound (化) is given by

$$
x_{c}^{P}=\left(1+D^{N} \operatorname{Max}\left\{\left|a_{l}^{*} a_{k}\right|\right\} / \sum_{k=-S}^{S}\left|a_{k}\right|^{2}\right)^{-1},
$$

where we denote by $\operatorname{Max}\left\{\left|a_{l}^{*} a_{k}\right|\right\}$ the maximum value of the product $\left|a_{l}^{*} a_{k}\right|(l \neq k)$ of the coefficients forming the general density matrix (2) and (16). On the other hand the largest eigenvalue $\bar{v}=\operatorname{Max}\left\{\left|a_{k}\right|^{2}\right\} / \mathcal{N}$ for the matrix $\tilde{\tilde{\rho}}$ in (11), give us, from (13) the entropic bound

$$
\begin{aligned}
x_{c}^{S} & =\left(1+\frac{D^{N} \operatorname{Max}\left\{\left|a_{l}^{*} a_{k}\right|\right\}}{\sum_{k=-S}^{S}\left|a_{k}\right|^{2}} \alpha\right)^{-1}, \\
\alpha & =\frac{\sum_{k=-S}^{S}\left|a_{k}\right|^{2}-\operatorname{Max}\left\{\left|a_{k}\right|^{2}\right\}}{(D-1) \operatorname{Max}\left\{\left|a_{l}^{*} a_{k}\right|\right\}} .
\end{aligned}
$$

For arbitrary values of $\left\{a_{k}\right\}$ we have $\alpha \leq 1$ and from (17) and (18) we see that the Peres criterion is in general more restrictive than the entropic one, i. e., $x_{c}^{P}<x_{c}^{S}$. For the standard Werner density matrix $\left(a_{-S}=\cdots=a_{S}=1\right)$ we have $\alpha=1$ and the result $x_{c}^{P}=x_{c}^{S}=1 /\left(1+D^{N-1}\right)$, that in this case coincides with the exact value of $x_{c}$ [28]. Like in our first application (14) and (15), whenever $x_{c}^{P}=x_{c}^{S}$, these values turn out to be the exact one. Equation (17) and (18) tell us that, whenever at least one of the components $a_{k}=0$ or at least a pair of coefficients exists such that $a_{k} \neq a_{k^{\prime}}$ in (16), $\alpha>1$ and these bounds are distinct. The coincidence of bounds $x_{c}^{P}=x_{c}^{S}$ happens only for the special case $a_{-S}=\ldots=a_{S}$.

It is important to notice that interpreting (2) with (16) as the coupling of an entangled state $\mid \tilde{\phi}>$ with the white spectrum environment, in order that the system stays entangled, any component of the environment should not exceed a fraction $r_{c}=\left(1-x_{c}\right) /\left(1+\left(D^{N}-1\right) x_{c}\right)$ of the component of the engineered entangled state $\tilde{\phi}$. 
Using the more restrictive bound $x_{c}^{P}$ (17) we obtain $r_{c}=\left(\sum_{k}\left|a_{k}\right|^{2}-\operatorname{Max}\left\{\left|a_{l}^{*} a_{k}\right|\right\}\right) / \operatorname{Max}\left\{\left|a_{l}^{*} a_{k}\right|\right\}$, that implies that $r_{c}$ decreases with the number of components where $a_{k} \neq 0$ in (16) and it is more difficult to keep the system entangled.

We have also studied other density matrices where the special entangled state $\mid \tilde{\phi}>$ are invariant under spatial translation on the spin ordering as well charge conjugation. In this case the results have to be carried numerically 30 and our results indicate concidences of the bounds $x_{c}^{P}$ and $x_{c}^{S}$ as the number of subsystems $N \rightarrow \infty$.

In conclusion we have shown that, although the Peres transposition method gives us more restrictive bounds than those coming from the nonextensive entropy, the application of both methods, that in general, at least numerically, is not a difficult problem, have the advantage of possibly providing, when the bounds $x_{c}^{P}$ and $x_{c}^{S}$ coincide, exact results, that are always of difficult derivation. Also we should remark that our analysis provides interesting results that should be useful for quantum computing, teleportation or cryptography. If the pure engineered entangled state $|\tilde{\phi}\rangle$ is the desired information we are processing $(x=1$ in (2) ), while time runs, the coupling with the environment (represented by $x$ in (2)) grows ( $x$ decreases) and the decoerence effect increases. As long as the system stays entangled, it might be possible to recover the desired information with appropriate correcting, distillation-like, procedures. Then, once we know the time dependence of $x(t)$, the bounds $x_{c}^{P}$ and $x_{c}^{S}$ will give us the time scale where the information should be processed.

One of us (C.T.) acknowledges enlightening remarks from R. Horodecki, P. Horodecki and M. Horodecki, as well as warm hospitality at the Schroedinger Institute, where this work was partially performed. The present effort has been partially supported by PRONEX, CNPq, and FAPERJ (Brazilian agencies).

[1] A. Einstein, B. Podolsky and N. Rosen, Phys. Rev. 47, 777 (1935).

[2] E. Schroedinger, Proc. Cambridge Philos. Soc. 31, 555 (1935).

[3] D.M. Greenberger, M. A. Horne and A. Zeilinger, in Bell's theorem, Quantum Theory, and Conceptions of the Universe, ed. M. Kafatos (Kluwer Academic, Dordrecht, 1989), p. 73; D.M. Greenberger, M. A. Horne, A.
Shimony and A. Zeilinger, Am. J. Phys. 58, 1131 (1990).

[4] A. Ekert, Phys. Rev. Lett. 67, 661 (1991).

[5] C. Bennett, G. Brassard, C. Crepeau, R. Jozsa, A. Peres and W.K. Wootters, Phys. Rev. Lett. 70, 1895 (1993).

[6] W.H. Zurek, S. Habib and J.P. Paz, Phys. Rev. Lett. 70, 1187 (1993).

[7] A. Peres, Quantum theory: Concepts and methods, (Kluwer, Dordrecht, 1993).

[8] S. Popescu, Phys. Rev. Lett. 72, 797 (1994).

[9] A. Barenco, D. Deutsch, A. Ekert and R. Jozsa, Phys. Rev. Lett. 74, 4083 (1995).

[10] R. Laflamme, C. Miquel, J.P. Paz and W.H. Zurek, Phys. Rev. Lett. 77, 198 (1996).

[11] M. Horodecki, P. Horodecki and R. Horodecki, Phys. Lett. A 210, 377 (1996).

[12] M. Horodecki, P. Horodecki and R. Horodecki, Phys. Lett. A 223, 1 (1996).

[13] A. Peres, Phys. Rev. Lett. 77, 1413 (1996).

[14] P. Horodecki, Phys. Lett. A 232, 333 (1997).

[15] M. Horodecki, P. Horodecki and R. Horodecki, Phys. Rev. Lett. 78, 574 (1997).

[16] S. Popescu and D. Rohrlich, Phys. Rev. A 56, R3319 (1997).

[17] M. Horodecki, P. Horodecki and R. Horodecki, Phys. Rev. Lett. 80, 5239 (1998).

[18] H. Touchette and S. Lloyd, Phys. Rev. Lett. 84, 1156 (2000).

[19] D. Bruss and A. Peres, Phys. Rev. A 61, 030301 (2000).

[20] C. Brukner and A. Zeilinger, Phys. Rev. A 63, 022113 (2001).

[21] S. Abe and A.K. Rajagopal, Physica A 289, 157 (2001).

[22] C. Tsallis, S. Lloyd and M. Baranger, Phys. Rev. A 63, 042104 (2001); C. Tsallis, P.W. Lamberti and D. Prato, Physica A 295, 158 (2001).

[23] M. Horodecki and P. Horodecki, Phys. Rev. A 59, 4206 (1999).

[24] S. Abe, Nonadditive measure and quantum entanglement in a class of mixed states of $N^{n}$-system, quantph/0104133.

[25] A.K. Rajagopal and R.W. Rendell, Robust and fragile entanglement of three qbits: Relation to permutation symmetry, quant-ph/0104122.

[26] B.M. Terhal, Detecting quantum entanglement, quant$\mathrm{ph} / 0101032$.

[27] C. Tsallis, J. Stat. Phys. 52, 479 (1988); Nonextensive Statistical Mechanics and its Applications, eds. S. Abe and Y. Okamoto, Series Lecture Notes in Physics (Springer-Verlag, Berlin, 2001). For a regularly updated bibliography see http://tsallis.cat.cbpf.br/biblio.htm.

[28] A. D. Pittenger and M. H. Rubin, Phys. Rev. A 62, $032312(2000)$.

[29] R. F. Werner, Phys. Rev. A 40, 4277 (1989).

[30] F. C. Alcaraz and C. Tsallis, to be published. 